\title{
EMPRESAS Y EMPRESARIOS: CAMINO AL ÉXITO Y PRODUCCIÓN DEL VALOR ECONÓMICO Y SOCIAL
}

Dra. Clara Caselli

\section{La idea de empresa}

Cuando pensamos en qué es una empresa, muchas veces pensamos en un conjunto de elementos (maquinarias, tecnologia, recursos financieros, recursos humanos, etc.) o en una serie de actividades (producción, logística, ventas, promoción, etc.). En realidad, la empresa no es una simple suma de personas, cosas, actividades; por lo tanto, no se puede entender su naturaleza sin percibir las relaciones dinamicas entre todos sus elementos y con el ambiente externo.

Desde el punto de vista de su definición, una empresa es el resultado de tres elementos:

- Una comunidad de personas que trabajan juntas para realizar una actividad económica. Esto es tan verdadero que vale aun en el caso que el empresario no quiera pensat en sus trabajadores como personas porque quiere explotar el trabajo mismo: siempre se trata de personas y no de rostros anónimos, que tienen sus caracteristicas humanas, sus expectativas, sus deseos, sus convencimientos, que juegan un rol importante al nivel de la estructura formal $\mathrm{y}$ de las dinámicas in formales.

El tema específico del cual se ocupa esta comunidad de personas debe ser algo que tiene que ver con la economia; este punto marca la diferencia con las demás activicades humanas: un grupo de personas que se encuentran para escuchat 


\section{Clara Casel11}

música, por ejemplo, no es una empresa (a menos que se trate de una actividad de organización de conciertos).

- Un objetivo común: el fin no es el simple provecho, el fin es construir algo útil para los que colaboran en la empresa y pata todos los que se relacionan con ella. Es decir, se tiene que buscar la satisfacción de las necesidades de los clientes y al mismo tiempo de los trabajadores, de los proveedores, de las instituciones financieras, de los socios, del estado, etc. Conseguir un beneficio no puede ser un objetivo final, sino un simple medio que garantiza la autonomia de la gestión (como veremos en el punto siguiente). Ganar no es tanto un fin sino un indicador de la eficiencia de la gestión. Si una empresa produce en pérdida no está creando valor, al contrario, lo está destruyendo y lo substrae de los demás, especialmente de las empresas eficientes: se trata de una falta evidente de responsabilidad social.

Además, una empresa no se justifica simplemente por su eficiencia: Ias empresas tienen que trabajar con eficacia, que se puede medir sólo como la satisfacción de los clientes y la de los actores sociales. Se entiende, por lo tanto, que la ganancia no puede ser un objetivo final: si una empresa gana mucho pero no cuida que sus trabajadores $-y$ también sus clientes, sus proveedores, los ciudadanos que viven cerca - estén satisfechos, la empresa no dura en el tiempo y se acaba después de unos años. El perfil del empresario es algo completamente distinto del perfil del especulador que sólo quiere ganar a corto plazo y luego no le importa si sale del mercado porque todo lo que tiene que hacer es buscar nuevas oportunidades de especulación.

- La capacidad de desarrollar su actividad de una manera autónoma, es decir, sin depender para su existencia de los bancos y de los demás que aportan capital de crédito, del estado, de la municipalidad, de otras empresas, etc.

La autonomía está relacionada con el deseo que la empresa dure en el tempo: para lograr este resultado se necesita evidentemente la capacidad de desarrollar autonomamente su propia dinámica de crecimiento y de autosustentamiento. La idea de duración en el tiempo es un aspecto lípico del perfil del auténtico empresario, que concibe su idea empresarial a partir de la observación de la 


\section{EMPRESAS Y EMPRESARIOS: \\ CAMINO AL ËXITO Y PRODUCCIŌN DEL VALOR ECONŌMICO Y SOCIAL}

realidad y la formula para moldear la realidad misma según su imagen ideal y con la perspectiva de dejar una huella en la historia economica y empresatial de supaís.

\section{El paradigma de la producción del valor}

La dignidad de cualquier iniciativa que tenga una naturaleza cconómica y lo que legitimz su existencia es la capacidad de producir valor en el ambiente económico y social: equé naturaleza tiene este valor? Porter afirma que todos los operadores buscan un valor en el desarrollo de suactividad y existe un sistema del valor porque el emptesario produce valor para si mismo y para todos los que entran en relación con su empresa. Lógicamente, la naturaleza de este valor es de ser un valor de compra, sin embargo, se trata también de un valor de uso. Además, está claro que existe una dialéctica entre valor de uso y valor de compra que se vuelve muy interesante en el contexto de la nueva economía de la información caracterizada por el hecho de que la producción cada vez metios se basa sobre elementos sólo materiales para asumir un fuerte carácter inmaterial.

Es interesante reflexionar sobre el paradigma conceptual de la producción de valor. La actividad de cualquier empresa inicia comprando valores de mercado para destinarlos a un uso bien identificado. Ya en esta fase hay un punto de creación de valor, porque puede ser que el comprador perciba un mayor valor (que se traduce en la convicción de haber concluido kun buen negocion). Después, el proceso productivo transforma estos valores y provoca su crecimiento, que normalmente es mucho más que la simple dinámica de los valores de compra, porque deriva del hecho que entran en juego babilidades, superioridades tecnológicas, que no siempre ténen un carácter tangible. Por último, la comparación con el mercado vuelve a set un momento en que se manifiestan diferentes percepciones del valor. La doctnna nos dice que el valor tiene que crecer de una fase a la otra: en caso contrario no se produciría valor económico sino desvalorización. Además, una empresa tiene que producir valor para si misma y para todos los actores con quienes entra en contacto. 


\section{Clara Caselli}

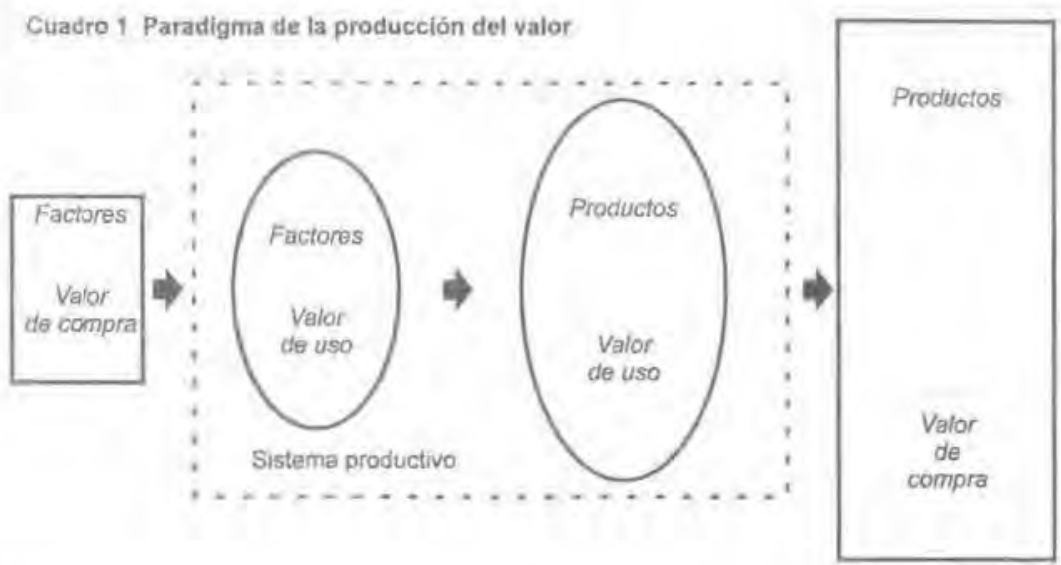

En sintesis el proceso se desarrolla según los siguientes pasos (cuadro 1):

1. Compra de factores de la producción: los factores se compran en un mercado del rabajo, de la materia prima, etc. y, por lo tanto, tienen un valor de compra, que es el precio de mercado.

2. Uso de los factores para producir bienes y servicios: en esta fase, los bienes tienen un valor de uso que depende del hecho de que el productor los está usando en un proceso productivo y se supone que este valor es más alto que el precio que ha pagado para conseguirlos (valor de uso de los factores $>/=$ valor de compra).

3. Transformación de los factores en productos terminados, que tienen para el productor un valor de uso más alto, porque él ha añadido trabajo, creatividad, satisfacción personal (valor de uso de los productos $>/=$ valor de uso de los factores productivos: por eso existen las empresas, porque se supone que tienen la capacidad de crear valor).

4. Venta de los productos en el mercado a un precio de mercado, que es un valor de compra para los compradores (precio de venta $>1=$ valor de uso de los productos para el empresario); a partir de este punto puede ser iniciado un nuevo ciclo de producción de valor por el comprador porque su valor de uso será más alto que el precio que ha pagado y de esta manera el circuito de 


\section{EMPRESAS Y EMPRESARIOS: \\ CAMINO AL EXITO Y PRODUCCIÓN DEL VALOR ECONÓMICO Y SOCIAL.}

producción del valor se vuelve social y se forma en la sociedad un sistema del valor que pone en relación con empresas, proveedores, consumidores, generando beneficios y satisfacciones en un entorno amplio.

El proceso que se acaba de describir puede ser llamado sparadigma de la producción del valon: por un lado tiene que ver con los aspectos económicos y Jucrativos $y$, por otro lado, incluye también aspectos subjetivos, que se refieren a la esfera humana y social.

\section{¿Cómo nace una empresa? El diseño de la fórmula empresarial}

Antes que nada se necesita una visión, que es el puerto al cual se quiere llegary es el resultado de la acción de pensar en el futuro a partir de la observación atenta de la realidad para imaginar una intervención en la realidad misma que pueda darle forma, descubriendo las oportunidades que se presenten, a partir de un ideal, de una kcultura», de unos juicios de valor sobre lo que es bueno, justo, útil, bello. La visión no es (y no debe ser) un sueño, sino algo que puede ser traducido en acción y puesto en marcha. Tiene que ser realizable, compartida, amplia, detallada (¿qué?, ¿cómo?, ¿cuándo?, ¿̨por qué?) y positiva.

Luego, se tiene que pensat en la misión de la empresa, que es la traducción de la visión, la ruta para llegar al puerto, en sus aspectos estratégicos.

Cuadro 2 La fórmula empresarial 1

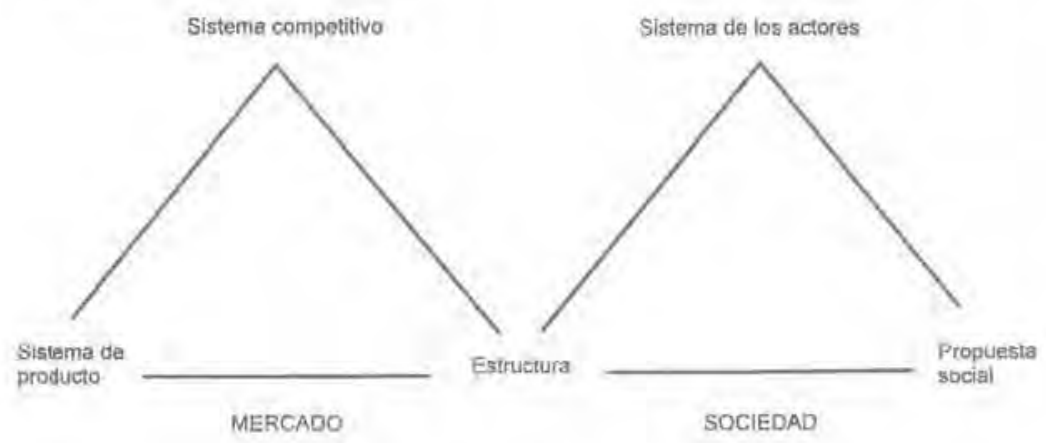




\section{Clara Casel11}

Para desarrollar esta dinámica, una metodología muy útil es el diseño de la "fórmula empresariab), es decir, un conjunto lógico de elementos que vuelven exitosa a una empresa. La fórmula se compone de dos aspectos que describen las diferentes lógicas que la empresa tiene que usar en su actividad. Gráficamente, usamos un esquema (cuadro 2) formado por dos triángulos: a la izquierda están las relaciones competitivas (el mercado) y a la derecha las relaciones no competitivas con todos los actores que entran en contacto con la empresa misma cooperando con ella (la sociedad). Una empresa tiene que imaginar su producto / servicio y el segmento de mercado al cual quiere llegar y construir su estructura de una manera coherente, pero al mismo tiempo tiene que conocer los sujetos que entran en relación no competitiva con ella (trabajadores, proveedores, empresas, socios, bancos, estado, etc.) y desarrollar su propuesta social hacia el sistema de los actores.

\section{Cuadro 3 La farmula empresarial 2}

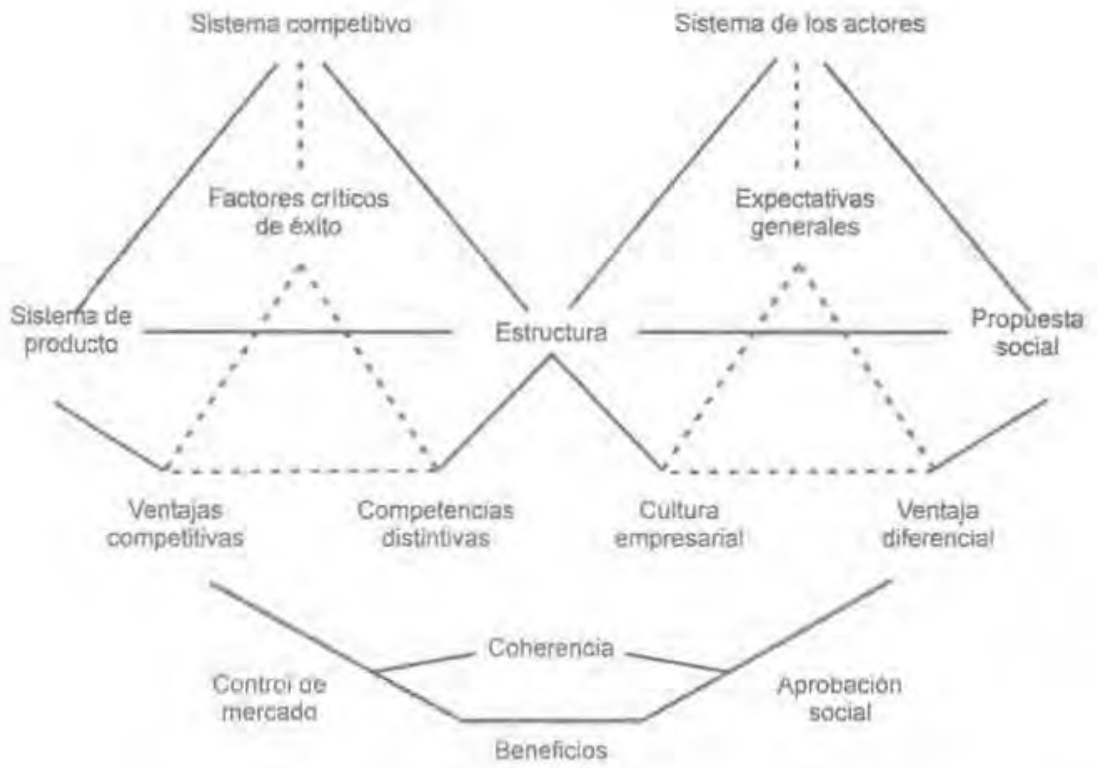




\section{EMPRESAS $Y$ EMPRESARIOS; \\ CAMINO AL EXITO Y PRODUCCION DEL VALOR ECONÓMICO Y SOCIAL}

Más analiticamente, la construcción de la förmula empresarial necesita unos pasos (cuadro 3):

1. Clientes y mercado

Primero, es necesario conocer el mercado para identificar el segmento al cual la producción está dirigida. El estudio del mercado presupone el análisis de la competencia que se encuentra en el mercado mismo, para comprender si la empresa puede obtener un espacio estable y rentable. Se trata de contestar preguntas de este tipo; ¿quién produce los mísmos productos o productos similares?, ¿dónde?, ¿a qué precio?, ¿hay un espacio para la empresa?, ¿cómo reaccionarín los competidores?, chasta dónde llega el ambiente competitivo?, ¿quiénes son los clientes objetivo?

2. Sisterna de producto

Se trata de identificar los elementos que componen la oferta de bienes y servicios de la empresa: de hecho, las empresas normalmente no producen simplemente productos sino un sistema complejo de productos y servicios, con un producto central (core product) y elementos accesorios, fundamentales para determinar la calidad ofertada y percibida por el cliente. Las preguntas son las siguientes: ¿qué se quiere producir?, ¿a quién se quiere vender?, ¿̨a qué precio?, ¿con qué nivel de calidad?

3. Estructura

La identificación de la estructura técnica y humana necesaria para implementar la idea empresarial es evidentemente muy importante: sin una estructura adecuada, la idea carecería de coherencia y realismo. Las preguntas son muy sencillas: ¿dónde se ubica la empresa?, ¿cuántos trabajadores se tienen que contratar y qué competencias tienen que poseer?, ¿cómo tienen que ser las maquinatias, la tecnologia, los muebles, etc.?

4. Sistema de los actores sociales

Se trata de todas las personas $y$ las instituciones que se relacionan con la empresa sin que tengan que ver con el sistema competitivo: empleados; proveedores; empresas con las cuales se estấn manejando acuerdos y alanzas productivas, tecnológicas, comerciales; bancos e instituciones financieras; socios que aportan capital: comunidad local; entidades públicas; etc. Es muy importante que la empresa identifique de una manera clara y completa el sistema de todos los actores que la rodean y de sus expectativas. 


\section{Clara Casel1i}

5. Propuesta soctal

La empresa tiene que formular una propuesta social dirigida a todos los actores económicos, políticos y sociales, que complementa y soporta la propuesta económica. Por ejemplo, los trabajadores tienen expectativas que se refieren al sueldo, al clima de trabajo, a las caracteristicas del trabajo mismo, etc; los proveedores quieren puntualidad de pago, estabilidad de relaciones, etc; los aliados exigen lealtad, continuidad y desarrollo de las relaciones; los bancos están interesados en el reembolso puntual del dinero y en el fortalecimiento de la estructura financiera de la empresa; los socios desean un dividendo al final de cada año; la comunidad local confia en un cierto tipo de presencia social de la empresa y en su colaboración en los proyectos de desarrollo; las entidades públicas como la municipalidad y el estado quieren cobrar impuestos, quieren también auspicios y colaboraciones, comportamientos éticos, etc.

El análisis de estos cinco primeros pasos permite la identificación de los elementos de la fórmula empresarial, pero no permite verificar su coherencia. Paa hacer esto, es necesario pasar a un nivel más sofisticado de analisis: por un lado, se tiene que verificar si el producto cumple con los requisitos que el mercado exige y si la empresa sabe realizar un producto a la altura de las expectativas; por otro lado, se tiene que evaluar si la propuesta que la empresa hace a los actores cumple con sus expectativas y con las características de la empresa. Esté proceso está descrito en el cuadro 3.

6. Factores críticos de éxito

Se trata de los elementos que determinan el éxito en un mercado (por ejemplo: calidad, precio, puntualidad en la entrega, etc.). Para escoger estos factores, la pregunta que hay que hacerse es la siguiente; ¿qué desean verdaderamente los clientes?, ¿cuál es el aspecto o los aspectos que les interesan más (se trata de escoger uno o dos factores, cuidando que no baya contradicción entre ellos: por ejemplo, es muy dificil dar calidad muy alta a un precio muy bajo)?, ¿cuál es el aspecto más importante para ganar con respecto a los competidores? (por ejemplo, el éxito de un restaurante especializado en pescado podria depender más que nada de la calidad del pescado mismo, el éxito de una consultoría más que nada de la competencia del consultor, etc.). 


\section{EMPRESAS Y EMPRESARIOS: \\ CAMINO AL ÉXITO Y PRODUCCIŌN DEL VALOR ECONÖMICO Y SOCIAL}

\section{Competencias diferenciales}

Se trata de la identificación de las habilidades exclusivas de la empresa, que la caracterizan y la diferencian de las demás empresas. Las preguntas para contestar son las siguientes: ¿cuáles son las competencias que más diferencian la empresa frente a los competidores?, ¿cuáles son las fortalezas más interesantes que la empresa posee? (por ejemplo, un buen chef, conocimientos tecnalógicos exclusivos, etc)

8. Ventajas competitivas

Si las competencias que la empresa tiene cumplen con los factotes críticos de éxito, se puede decir que la empresa tiene ventajas competitivas auténticas. Se tratará de ver también si son durables en el tiempo, es decir, si pueden resistir los ataques de los competidores.

9. Expectativas generales

Se trata de entender bien lo que los actores sociales esperan exactamente de la empresa y qué les interesa más, es decir, se tiene que establecer una jerarquia entre las expectativas.

10. Cultura empresarial

Son los valores de la organización, compartidos por todos; forman el clima de la organización que permite la realización de las aspiraciones de los actores. Para identificar cual es la cultura de una organización puede ser útil entrevistar a sus jefes, pero mucho más se puede descubrir simplemente observando cómo la empresa actúa, el tipo de relaciones entre los trabajadores, el tipo de trato hacia los clientes, etc. El punto fundarmental es entender si la empresa sabe darse cuenta de las expectativas y desea entrar en sintonía con ellas tomando inciativas adecuadas.

11. Ventajas diferenciales

Si la empresa sabe entender las exigencias de los actores y desea contestar positivamente, la propuesta social se concretizará en una serie de ventajas que los actores tendrân eritrando en contacto con la empresa; en otras palabras, son las ventajas que los actores logran alcanzar comprometiéndose con la empresa y aceptando colaborar con ella; se trata de un conjunto de aspectos que genetan en los actores mismos el convencimiento de que relacionarse con aquella empresa cumple con las expectativas mucho mảs que con cualquier otra. 


\section{Clara Casel11}

\section{Coherencia}

El análisis que se ha hecho hasta este punto permite dar una evaluación muy puntual del nivel de coherencia de la fórmula empresarial adoptada: al final, tiene que existir una coherencia cualitativa y cuantitativa entre los diferentes aspectos de la formula empresarial Las preguntas son: ctodos los elementos de la fórmula empresarial pueden existir contemporáneamente?, dla propuesta económica y la propuesta social son compatibles?

13. Control del mercado

Si hay coherencia entre los elementos del triángulo izquierdo del cuadro 2 , Ia empresa toma una posición de supremacía en el mercado, que le permite tener poder de convocatoria y capacidad de ganar y de crecer, satisfaciendo las necesidades de sus clientes. En otras palabras, logra controlar y dominar sumercado.

14. Aprobación social

Al mismo tiempo, si hay coherencia entre los elementos del triángulo derecho del cuadro 2 , se logra conseguir un consenso social vasto en un ambiente tranquilo y protegido.

15. Generación de beneficios

A largo plazo, la empresa logra conseguir beneficios que le permiten autosustentarse y se mantiene sólida y segura en el medio de su ambiente económico y social.

\section{La coherencia cuantitativa}

La coherencia cualitativa puede ser verificada a nivel de correspondencia entre los elementos de la fótmula empresarial y requiere un análisis estratégico, mienteas que la coherencia cuantitativa necesita un análisis económico y financiero. El cuadro 2 puede ser leido desde este punto de vista: en la parte izquierda se generan los ingresos (ventas de productos y servicios) y en la parte derecha se originan los costos (salarios, compras de materia prima, gastos de producción y comercialización, intereses, impuestos, comisiones, etc.): la dinámica de los ingresos y de los costos es la spelículas que describe la actividad de la empresa durante un cierto tiempo (por ejemplo in año) y permite, incluyendo todos los 


\section{EMPRESAS Y EMPRESARIOS: \\ CAMINO AL EXITO Y PRODUCCIÓN DEL VALOR ECONONMICO Y SOCIAL}

elementos (también los que no tienen uma naturaleza financiera) y haciendo todos los ajustes contables necesarios, ta evaluación del beneficio o de la pérdida conseguidôs. Además de la upelículas que sintetiza la actividad de la empresa en el tiempo, es posible etomar la foton del estado del activo y del pasivo, para tener un cuadro del capital invertido (capital fijo y capital de trabajo) y de sus modalidades de cobertura. Los dos cuadros que se pueden construir dan una idea de la capacidad de la empresa de mantencrse autónoma en el tiempo y miden la adecuación de los recursos y la flexibilidad de la estructura.

El aspecto de la flexibilidad merece unas consideraciones más: un aspecto fundamental se refiere a las decisiones sobre las modalidades de actuar de la empresa que influyen en la adquisición y el uso de los recursos. En otras palabras, con respecto a las actividades que la empresa quiere desarrollar se pueden tomar tres decisiones diferentes, es decir, (hacen, ucompran,, "cooperan. Si la decisión es hacer directamente una actividad, son necesarias inversiones y se originan gastos que deben ser justificados por un alto margen, al mismo tiempo la estructura se vuelve inevitablemente más rigida y los costos fijos son más altos. Si la decistón es comprar, la flexibilidad es máxima, se reducen los costos y se simplifica la producción, pero se pierde el control directo de las actividades realizadas afuera y se reducen los márgenes. Por fin, la cooperación es una alternativa muy interesante porque reduce los costos y permite flexibilidad sin perder el control de la actividad, aun exigiendo la capacidad de colaborar entre sujetos diferentes, que es dificil pero desafiante porque solicita la capacidad de pensar en la producción del valor como un fenómeno no simplemente individual sino social.

\section{Un nuevo tipo de sujeto cconómico: la empresa no lucrativa}

En los últimos años, muchas investigaciones en el campo de la economía y administración han dedicado atención al terna de las empresas no lucrativas. En realidad, estas empresas existen en fodo el mundo hace muchisimos años, a veces preceden el nacimento de los estados modernos como en el caso de las grandes obras de acogida y de asistencia a los pobres que nacieton a partir de la experiencia de la Iglesia ya en la Edad Media o también como en el caso de las grandes 
universidades en sus origenes. Dos son los motivos del interés y de la atención creciente que las empresas no lucrativas están recibiendo: primero, la crisis del estado de bienestat (welffare state) en los paises desarrollados que ha hecho descubrir todo el valor de estas formas de organización de la producción, especialmente en el sector de los servicios a la persona, donde estas empresas saben organizar servicios de calidad a costo bajo; segundo, la creación de nuevos puestos de trabajo por este sector en un periodo histórico que se caracteriza por el insuficiente desarrollo de las oportunidades de empleo en los sectores tradicionales de la producción.

Hasta ahora, la relevancia del fenómeno no ha sido muy investigada en los países en desarrollo, esencialmente por falta de informaciones estadisticas adecuadas y. de conciencia de la importancia de este sector para fomentar crecimiento económico y social. Sin embargo, esto no quiere decir que no se encuentren empresas y organizaciones no lucrativas en estos países: al contrario, la experiencia enseña que las experiencias son muchas y muy articuladas; talleres, comedores populares, postas médicas, colegios, cooperativas de producción y distribución, etc.

Antes que nada, es importante subrayar que la empresa no lucrativa no deja de ser una empresa por el hecho de no apuntar al provecho.

Si retomamos el paradigma de la producción del valor en el caso de las empresas no lucrativas, nos damos cuenta de que el sistema de desigualdades descrito en el cuadro I puede presentar diferencias muy considerables: los factores de la producción se pueden comprar fuera de la lógica del mercado (y no se tratará de un "buen negocion porque simplemente se ha dado una ruprura del paradigma en consecuencia del papel que juegan los elementos no económicos en sentido estrecho). Lo mismo pasa cuando la negociación de los productos / servicios adopta precios que no son de mercado: en este caso la desigualdad cambia de dirección sin que se pueda decir que el proceso productivo es ineficiente.

Una empresa no lucrativa se caracteriza por dos aspectos; el lucro no es el fin principal y roda la actividad tiene unia ocientación social (social benefít). Tratando de profundizar esta definición, se puede decir que la caracteristica principal es que los valores de uso son más importantes que los valores de compra, de mercado, En otras palabras, lo que las cosas asonv es más importante que el precio que mide su valor: por un lado se trata evidentemente de estar en el mercado respetando sus 


\section{EMPRESAS Y EMPRESARIOS: \\ CAMINO AL ËXITO Y PRODUCCIÓN DEL VALOR ECONÓMICO Y SOCIAL}

reglas pero, por otro lado, es muy evidente la presencia y la importancia fundamental de una dinámica de iscosas que no tienen precion.

Esta concepción tiene consecuencias sobre la formulación de las líneas estratégicas y el diseño de la fórmula empresarial: el tríngulo derecho es mucho más importante y también la lógica de cooperación que se desatrolla entre los actores sociales: en realidad, la fórmula empresarial en este caso siempre es una fórmula social que subraya el dinamismo de innovación en el ambiente y la capacidad de construit alianzas (la red del valor social). Sin embargo, es importante añadir que no se quita nada a la exigencia de una gestión empresarial eficiente, que es imprescindible, especialmente, a partir de un cierto estadio de desarrollo de la empresa no lucrativa.

\section{¿Qué significa éxito para la empresa?}

Después del análisis de las características de las empresas lucrativas y no lucrativas, es interesante desarrollar unas consideraciones conclusivas sobre las condiciones del éxito de cualquier tipo de empresa.

El convencimiento que más ha sido subrayado en este articulo es que tener éxito no quiere decir conseguir un alto tivel de ganancia. En todas las empresas, también las no hucrativas, el provecho es un simple indicador de eficiencia de la gestión que revela la capacidad de autosustentarse y generar valor en el tiempo, es decir, de durar manteniendo su autonomia.

El provecho como fin último de la actividad de la empresa ha perdido valor y sigue perdiéndolo. Por ejemplo, en los periodos de crisis económica, muchas empresas no obtienen beneficios sino pérdidas; a veces una empresa que pertenece a un grupo no consigue una ganancia, pero genera un valor a nivel de agrupación; también hay empresas que no ganan directamente sino indirectamente porque producen economias externas positivas para la sociedad (etr este caso, que se encuentra en muchas empresas no hucrativas, se necesitaria la redacción de un balance social para darse cuenta de la contribución a la producción del valor). Se podrian hacer muchos ejemplos de este tipo: en realidad ninguna empresa exitosa está orientada hacia la simple rentabilidad económica. 


\section{Clara Caselli}

El éxito de una empresa está apoyado sobre tres diferentes ejes. Uno es de tipo económico y se refiere a la capacidad de desarrollar la actividad manteniendo los costos por debajo de los ingresos; el segundo es un éxito competitivo y se refiere al crecimiento de los clientes, de su grado de satisfacción, de los ingresos conseguidos; el tercero es el éxito social, es decir, la satisfacción de los empleados, de los proveedores, de los bancos, etc. Un éxito económico que se acompaña a una cuota reducida del mercado y a la insatisfacción de los actores revela una actividad especulativa de corto plazo; un éxito competitivo con bajos márgenes señala la dificultad de conseguir una posición estable de dominancia del mercado; un éxito social sin conseguir buenos resultados en materia de competitividad y rentabilidad revela una posición abstracta y soñadora.

En todo lo que se ha dicho hasta este punto, hay que rescatar y subrayar la palabra «actob) (o protagonista): si se encuentran actores el mercado no es un mecanismo anónimo gobernado por una "mano invisible), sino una posibilidad de encuentro y confrontación entre personas y empresas que tienen una identidad. De esta manera se afirma una concepción de la economía y de la sociedad articulada, con una riqueza de iniciativas donde cada sujeto es responsable hacia todos los demás, en primer lugar por la originalidad de su misión.

Un actor tiene su visión y su misión, y formula una propuesta que es al mismo tiempo economica y social, es decir, con su dinamismo en el ambiente produce innovación social en el sentido que crea en la sociedad algo nuevo, algo especial, que faltaría completamente sin su presencia. Se entiende, por la tanto, que en realidad la fórmula empresarial es, por su naturaleza, una fórmula social, y se entiende tambièn que cualquier discurso de ética de la empresa tiene que partir de este punto, porque el problema no consiste en retomar o recuperar valores abstractos sino en producir valor y participar responsablemente a la construcción de la red del valor.

Estas ideas generales tienen su validez en cualquier contexto económico, se trate de un pais tico o de un pais que tiene que desarrollarse. La fórmula empresarial usada para fomentar el crecimiento de la economía y en particular de las Pymes en el tercer mundo tiene que tomar en cuenta todos los aspectos que se han descrito antes, subrayando más todos los elementos que hacen progresar el nivel de los recursos humanos y de la responsabilidad social, porque éste es el punto de partida para romper ć́rculos viciosos que no dejan crecer sujetos protagonistas de su 


\section{EMPRESAS Y EMPRESARIOS: \\ CAMINO AL ÉXITO Y PRODUCCIÓN DEL VALOR ECONÓMICO Y SOCTAL.}

desarrollo e iniciar circulos virtuosos que sepan poner en marcha sinergias entre el sector pública, las organizaciones internacionales, las empresas privadas y las iniciativas no lucrativas y de voluntariado.

La experiencia nos dice que se trata de algo posible: por eso los dos atticulos siguientes usan Ta metodología descrita para proponer dos casos de éxito en el Perú.

\section{Bibliografia}

AIROLDI G., BRUNETTI G., CODA V., 1989, Lezioni di economia aziendale, Bologna, Il Mulino.

BORZAGA C, SANTUARI, , 2000,

The Innovative Trends in the Non-profit Sector in Europe: the Emergence of Social Entrepreneurship, Conference Paper OECD/LEED Forum on Social Innovations.

CASELIC., 1998,

kllnon prō̄t: aspetti aziendalis, Persone \&: Imprese, N. 2 .

CASEILI C., 2000a, "Non profit: una totale dignitá di azienda", Persone, Imprese \&. Istiruzioni, N." I.

CASELLI C., 2000b, "Oltre la finanza etican, Persone, Imprese \& Istituzioni, N.e3.

CODA V, 1984, La valutazione della formula mprenditoriale, Sviluppo e Organizzazione, N.82

SALAMON L. M., ANHEIER H. K, 1994a, $\mathrm{Il}$ settore nonprofit in un confronto internazionale: profili e tipi, Universitá Cattolica del sacro Cuore, Centro di ricerche sulla cooperazione, Quadernioccasionali, N.3.3.

SALAMON L. M., ANHEIER H. K, 1994b, Il settore nonprofit in una prospettiva comparata. Una panoramica, Università Cattolica del sacro Cuorc, Istirito per la ricerca sociale, Quaderni occasionali, N,"6.

VITTADINI G. (a cura di), 1997, II non profit dimezzato, Etaslibri, Milano.

VITTADINI G. (a cura di), 1998; Sussidiarietá. La riforma possibile, Etaslibri, Milano. 